\title{
Occurrence Rates from Direct Imaging Surveys
}

\author{
Brendan P. Bowler and Eric L. Nielsen
}

\begin{abstract}
The occurrence rate of young giant planets from direct imaging surveys is a fundamental tracer of the efficiency with which planets form and migrate at wide orbital distances. These measurements have progressively converged to a value of about $1 \%$ for the most massive planets $\left(\approx 5-13 M_{\text {Jup }}\right)$ averaged over all stellar masses at separations spanning a few tens to a few hundreds of AU. The subtler statistical properties of this population are beginning to emerge with ever-increasing sample sizes: there is tentative evidence that planets on wide orbits are more frequent around stars that possess debris disks; brown dwarf companions exist at comparable (or perhaps slightly higher) rates as their counterparts in the planetary-mass regime; and the substellar companion mass function appears to be smooth and may extend down to the opacity limit for fragmentation. Within a few years, the conclusion of second-generation direct imaging surveys will enable more definitive interpretations with the ultimate goal of identifying the dominant origin of this population and uncovering its relationship to planets at smaller separations.
\end{abstract}

\section{Introduction}

Direct imaging is the foremost method to study giant planets at wide orbital distances beyond about $10 \mathrm{AU}$ and complements radial velocity, transit, microlensing, and astrometric discovery techniques which probe smaller separations closer to their host stars. High-contrast imaging from the ground makes use of adaptive optics systems that largely operate at near-infrared wavelengths, making this method most

Brendan P. Bowler

Department of Astronomy, The University of Texas at Austin, Austin, TX 78712, USA, e-mail: bpbowlereastro.as.utexas.edu

Eric L. Nielsen

Kavli Institute for Particle Astrophysics and Cosmology, Stanford University, Stanford, CA 94305, USA, e-mail: enielsenestanford.edu 
sensitive to thermal emission from massive, warm giant planets. As a result, direct imaging surveys predominantly focus on the closest and youngest stars before planets have cooled to faint luminosities and low temperatures. This makes target samples for imaging surveys unusual compared to other planet detection methods, which predominantly focus on old (several Gyr) field stars with lower activity and jitter levels.

In addition to individual discoveries, high-contrast imaging surveys deliver statistical constraints on the occurrence rates and demographics of giant planets at large orbital distances. The frequency of giant planets and their mass-period distributions provide valuable information about the efficiency of planet formation and migration to large separations, and have been used to both guide and test giant planet formation routes. Nearly two dozen young planets have now been imaged with inferred masses as low as $2 M_{\text {Jup }}$ and separations spanning a large dynamic range of $\approx 10$ $10^{4} \mathrm{AU}$ (Figure 1). Several formation routes can explain the origin of gas giants at these unexpectedly wide separations: core and pebble accretion (Lambrechts and Johansen 2012), disk instability (e.g., Durisen et al.|2007, Kratter and Lodato 2016), turbulent fragmentation (Bate et al.|2003), and planet-planet scattering (Veras et al. 2009). In principle these pathways should imprint unique signatures on the resulting occurrence rates, mass-period distributions, and three-dimensional orbital architecture of exoplanets, although these are challenging to discern in practice due to the low incidence of widely-separated planets, a diversity of theoretical predictions, the the potential for subsequent migration to occur, and the difficulty of constraining orbital elements for ultra-long period planets (e.g., Blunt et al. 2017). One of the emerging goals for direct imaging is to untangle the dominant formation route(s) of this population, which is best accomplished in a statistical fashion with expansive high-contrast imaging surveys.

The largest-scale surveys exploiting extreme adaptive optics systems like the Gemini Planet Imager (GPI) and the Spectro-Polarimetric High-contrast Exoplanet REsearch (SPHERE) are currently underway. These second-generation instruments utilize integral field spectrographs for speckle suppression through spectral differential imaging and achieve unprecedented on-sky contrasts at small $\left(<1^{\prime \prime}\right)$ angular separations using coronagraphs. These results will eventually be merged with firstgeneration surveys to provide statistics of giant planets for samples exceeding one thousand young stars. In the future, the James Webb Space Telescope, the Extremely Large Telescopes, and space-based telescopes with coronagraphs working in the optical like WFIRST (and perhaps HabEx or LUVOIR) will expand this parameter space to lower masses, closer separations, and older ages.

Calculating occurrence rates for direct imaging surveys is a unique challenge. Surveys yield few detections, which means the underlying planetary mass-semimajor axis distributions are poorly constrained. This differs from other methods where detections are plentiful and the intrinsic functional form of the planet size, mass, and period distributions can be precisely measured. Instead, several assumptions must be made - or details ignored - about the underlying demographics and the way in which planets may scale with stellar mass. 

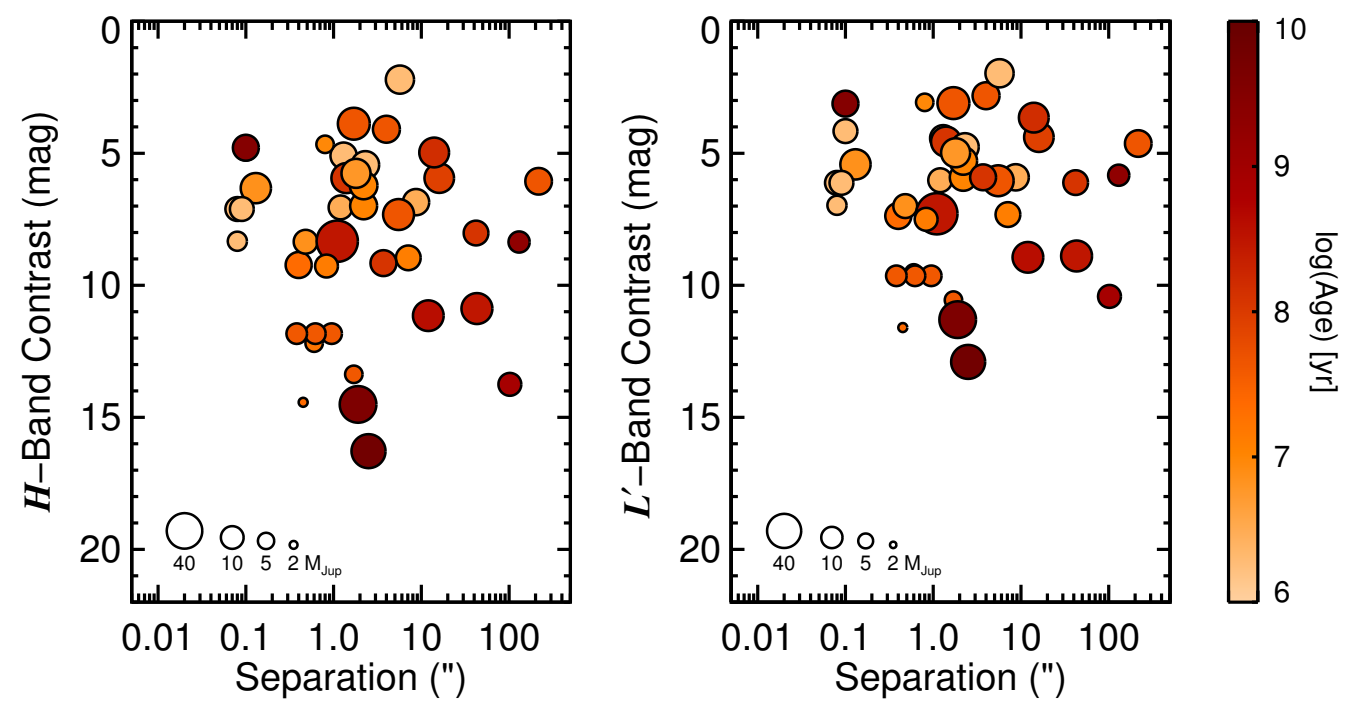

Fig. 1 Imaged companions with masses near or below the deuterium-burning limit. Objects are from Bowler (2016) and are supplemented with recently-discovered companions from Bowler et al. (2017) and Chauvin et al. (2017). Photometry represent actual measurements or values taken from the hot-start evolutionary models of Baraffe et al. (2015) based on the inferred companion mass and age, here represented by the size and color of the symbol.

Here we provide an overview of the methods and assumptions most widely adopted to calculate occurrence rates from direct imaging surveys, as well as a summary of observational results from the largest adaptive optics imaging surveys and meta-analyses carried out to date in the brown dwarf $\left(\approx 13-75 M_{\text {Jup }}\right)$ and planetary $\left(<13 M_{\text {Jup }}\right)$ mass regimes.

\section{Calculating Occurrence Rates}

High-contrast adaptive optics imaging is sensitive to angular scales spanning an inner working angle, often set by the size of a coronagraph, and an outer working angle limited by the field of view of the detector. The primary products of an observation is astrometry and relative photometry of point sources as well as a contrast curve in $\Delta$ mag (flux ratio) which defines the sensitivity of an observation at some statistical threshold. Point sources may be bound companions or (more often the 
case) background stars, which are distinguished via their relative motion or colors. Transforming high-contrast imaging observations to giant planet frequencies relies on a series of assumptions about the brightness of planets in particular filters as well as the underlying shape of the planet mass, separation, and eccentricity distributions. Here we provide an outline of the typical end-to-end procedure carried out by most surveys:

- A survey is designed and first epoch observations are acquired.

Direct imaging surveys have been continually increasing in size and sensitivity over the past 15 years. Target samples have evolved from several dozen stars to several hundred in size, although this is ultimately limited by the number of nearby young stars. Similarly, representative limiting contrasts have improved from $\approx 10 \mathrm{mag}$ at $0.5^{\prime \prime}$ (Biller et al. 2007) to $>14 \mathrm{mag}$ with second-generation extreme AO systems (e.g., Samland et al. 2017). Recent surveys generally boast a strategic advantage over previous work by targeting newly-identified nearby young stars, taking advantage of novel speckle suppression methods, or probing closer inner working angles with innovative coronagraph designs. The most successful surveys with the highest impacts are those that aim to answer specific science questions with clear and measurable goals. Reconnaissance work is an important component to designing an efficient survey, for example by vetting close visual binaries using adaptive optics on small telescopes, or by reassessing stellar ages to prioritize the youngest stars. First-epoch observations are acquired for all targets with typical integration times of about an hour each.

- Speckle subtraction is carried out, point sources are identified, and limiting contrasts are calculated.

Several methods for PSF subtraction have been developed over the past decade. Their aim is largely the same: to remove the static diffraction pattern and residual speckle noise from the host star while minimizing self-subtraction from real point sources. The most widely-used algorithms are variations of the Locally-Optimized Combination of Images (LOCI; Lafrenière et al. 2007b) - a least-squares approach which aims to represent science frames as linear combinations of reference images — and Karhunen-Loève Image Projection (KLIP; Soummer et al. 2012) — a technique that utilizes principal component analysis to identify and remove quasistatic PSF structure. For integral field units and dual-channel imagers, PSF subtraction takes advantage of the wavelength-dependency of speckles, which are magnified (and better-corrected) at longer wavelengths. An incomplete list of advances, modifications, and upgrades to these algorithms can be found in Marois et al. (2010); Pueyo et al. (2012), Brandt et al. (2013), Currie et al. (2014), Marois et al. (2014); Wahhaj et al. (2015), Cantalloube et al. 2015.,Wang et al. (2016), Pueyo (2016), and Ruffio et al. (2017).

Residual speckle noise can resemble planets, so automated point source identification is ideal to minimize biases and develop quantitative, measurable, and reproducible results based on detection threshold metrics like signal-to-noise ratio or reduced $\chi^{2}$ value (Wahhaj et al. 2013a). Reporting contrast curves for null results and detections alike are critical for deriving statistical constraints. Approaches involving injection-recovery of point sources as a function of separation, contrast, and 
azimuthal angle offer a robust means of deriving sensitivity limits. Special care must be taken when calculating confidence levels at small separations where the number of independent resolution elements diminishes (Mawet et al. 2014). Reporting the fractional field of view coverage (from $100 \%$ sensitivity down to $0 \%$ ) is also recommended and ideally should be incorporated in the survey statistical analysis. More recently, Jensen-Clem et al. (2018) propose a more formal strategy in the form of performance maps, which are based on signal detection theory and incorporate the true positive fraction of planets, the false positive fraction, and detection thresholds (see also Ruffio et al.2017).

- Second-epoch observations are taken to determine the nature of point sources.

Second-epoch observations are usually required to distinguish between background stars and comoving, gravitationally-bound companions, which may also exhibit some orbital motion. This assumes that background stars have zero (or negligible) proper motion compared to typical astrometric uncertainties (several milliarcseconds). However, Nielsen et al. (2017) showed that the close comoving point source near HD 131399 (Wagner et al.2016) may instead be an unfortunate example of a background star with a similar proper motion. Even with second epochs, targets with low proper motions can appear to be comoving and statistical false alarm probabilities should regularly be reported. On the other hand, if colors or spectral information are acquired during first-epoch observations then false alarm probabilities can be calculated using stellar space densities of ultracool dwarfs to statistically validate late-type companions (e.g., Macintosh et al.|2015).

Given limitations of finite telescope time and the fact that new candidate planets can be revealed in follow-up imaging means that surveys often end without having determined the nature of some candidate planets. Since these untested point sources will be bound planets or background stars, it is imperative that these sources be documented, reported, and treated in a consistent manner when calculating occurrence rates. A new Direct Imaging Virtual Archive (DIVA) database has been created to assemble processed images, published contrast curves, and candidate companions (see Vigan et al.2017 for details).

- Sensitivity limits in planet mass and separation are determined using evolutionary models.

Monte Carlo simulations of synthetic planets on random orbits offer a straightforward approach to transform contrast curves into sensitivity maps in mass and separation - that is, the probability that a planet with a given semi-major axis and mass would have been detected in a given observation or set of observations. Uncertainties in a star's age and distance can naturally be folded into this type of analysis. Another advantage of the Monte Carlo method is that if a target has been observed on multiple occasions spanning more than one epoch, these contrast curves can readily be used to jointly constrain the properties of potential companions. Circular orbits are often assumed, but the underlying orbital eccentricities of the simulated planets can alter the final results. This requires the use of evolutionary models to transform a planet's mass and age into an apparent brightness in a particular filter. Commonly used "hot-start" models which ignore radiative losses during planet assembly include Cond models (Baraffe et al. 2003) and BT-Settl models (Baraffe 
et al. 2015). "Cold-start" prescriptions will produce comparatively pessimistic limiting masses as they take into account dissipative accretion luminosity during the epoch of planet formation (e.g., Fortney et al. 2008)

- Occurrence rates are calculated assuming an underlying planetary massperiod distribution.

A universal goal of direct imaging surveys is to constrain both the underlying shape and overall occurrence rate of giant planets in order to evaluate the demographics of giant planets, measure the efficiency of planet formation, and test for correlations with stellar parameters. Over two dozen companions near and below the deuterium-burning boundary have been discovered with direct imaging (e.g., Bowler 2016), but only a handful of these were found in published surveys so the intrinsic underlying distribution of giant planets at large separations is not well known.

What is clear is that planetary-mass objects reside in a wide range of contexts: they have been found orbiting high-mass stars (Marois et al. 2008, Lagrange et al. 2010; Macintosh et al. 2015), low-mass stars (Bowler et al. 2017), brown dwarfs (Chauvin et al. 2005: Todorov et al.2010), nested within debris disks (e.g., Rameau et al. 2013b), multiple systems (Delorme et al. 2013), free-floating without a host (e.g., Liu et al. 2013), and even as binary pairs themselves (Best et al.|2017). These companions exist over a wide range of parameter space spanning five orders of magnitude in orbital distance and merge with the population of higher-mass brown dwarf companions, making their mass, separation, and eccentricity distributions difficult to unambiguously characterize.

With only limited knowledge of the planet distribution function, and without compelling physical motivation for an alternative model at wide separations (although see Meyer et al. 2017), a smooth double power law in mass and semimajor axis is commonly used:

$$
\frac{d^{2} N}{d M d a} \propto M^{\alpha} a^{\beta}
$$

An inner and outer cutoff in semi-major axis can be applied to ensure the distribution is constrained over the interval in which imaging data exists (typically between $\approx 5-$ $500 \mathrm{AU}$ ). Close binaries are generally avoided or removed from these samples. The planet distribution function can also be modified to take into account multiple planet systems like HR 8799 (e.g., Wahhaj et al. 2013b). The absence of similar systems strongly implies that the probability of a star hosting a planet depends on whether another planet exists in the system; that is, they are not independent events (see, e.g., Brandt et al. 2014b) .

Information about the eccentricity distribution is slowly trickling in via orbit monitoring of imaged planets. Except for the putative companion to Fomalhaut, which has $e=0.8 \pm 0.1$ (Kalas et al. 2013: Beust et al. 2014), all imaged planets with orbit constraints are inconsistent with high eccentricities: HR 8799 bcde and $\beta$ Pic b are all on low-eccentricity orbits with $e<\sim 0.3$ (e.g., Macintosh et al. 2014; Nielsen et al. 2014; Wang et al. 2016, Konopacky et al. 2016a; Wertz et al. 2017), 51 Eri b is only marginally constrained at $e<0.7$ (De Rosa et al. 2015), and HD $95086 \mathrm{~b}$ has $e<0.44$ (Rameau et al. 2016). With the exception of $\beta$ Pic b, these planets have 
been observed for significantly less than an orbital period, so in most cases their eccentricities are poorly constrained.

Detections and nondetections can be treated as Bernoulli trials and occurrence rates can be calculated with binomial statistics by taking into account the planet sensitivity maps (e.g., Lafrenière et al. 2007a, Nielsen and Close 2010, Bowler et al. 2015). Alternatively, planet frequencies can be calculated by integrating under the fitted planet distribution function (e.g., Brandt et al. 2014b; Clanton and Gaudi 2016). In general, a decision must be made about how to treat point sources that have not been confirmed or refuted as planets. One possibility is to truncate the contrast curve for those particular targets at some threshold above the candidate companion to exclude that information from the survey (Nielsen et al. 2013, Bowler et al.|2015, Meshkat et al. 2017).

Bonavita et al. (2013) have created a publicly-available package to carry out statistical analysis of direct imaging surveys- Quick Multi-purpose Exoplanet Simulation System (QMESS) - which offers a computationally fast, flexible, grid-based statistical tool for measuring occurrence rates (see also the Monte Carlo-based package from Bonavita et al. 2012). Brandt et al. (2014b) offer an alternative, analytic framework to transform a set of survey observations into statistical constraints on the companion frequency and mass function.

\section{Occurrence Rate of Giant Planets on Wide Orbits}

The frequency of massive planets on wide orbits has progressively come into focus over the past decade (Figure 2, see also Bowler 2016 for a detailed overview). Giant planets at large orbital distances are unquestionably rare, especially in relation to lower-mass planets being found with radial velocity and transit methods. This dearth of widely-separated gas giants gradually became apparent with the conclusion of large-scale surveys carried out at 8-10-meter class facilities over the past 15 years with a wide range of instruments, most notably by Biller et al. (2007), Lafrenière et al. (2007a), Heinze et al. (2010), Chauvin et al. (2010), Vigan et al. (2012), Rameau et al. (2013a), Yamamoto et al. (2013), Janson et al. (2013), Biller et al. (2013), Nielsen et al. (2013), Wahhaj et al. (2013b), Brandt et al. (2014a), Bowler et al. (2015), Chauvin et al. (2015), Galicher et al. (2016), Lannier et al. (2016), Uyama et al. (2017), and Naud et al. (2017).

Merging discoveries and detection limits from published surveys into ever-larger samples has become standard practice in order to improve the precision of occurrence rate measurements. These large meta-analyses carry the most statistical weight and are necessary to test for potential correlations with stellar properties and environmental context, which are ultimately expected to yield clues about planet formation and migration pathways. The following results are based on compilations of several surveys and assume hot-start evolutionary models to infer planet masses and sensitivities. 


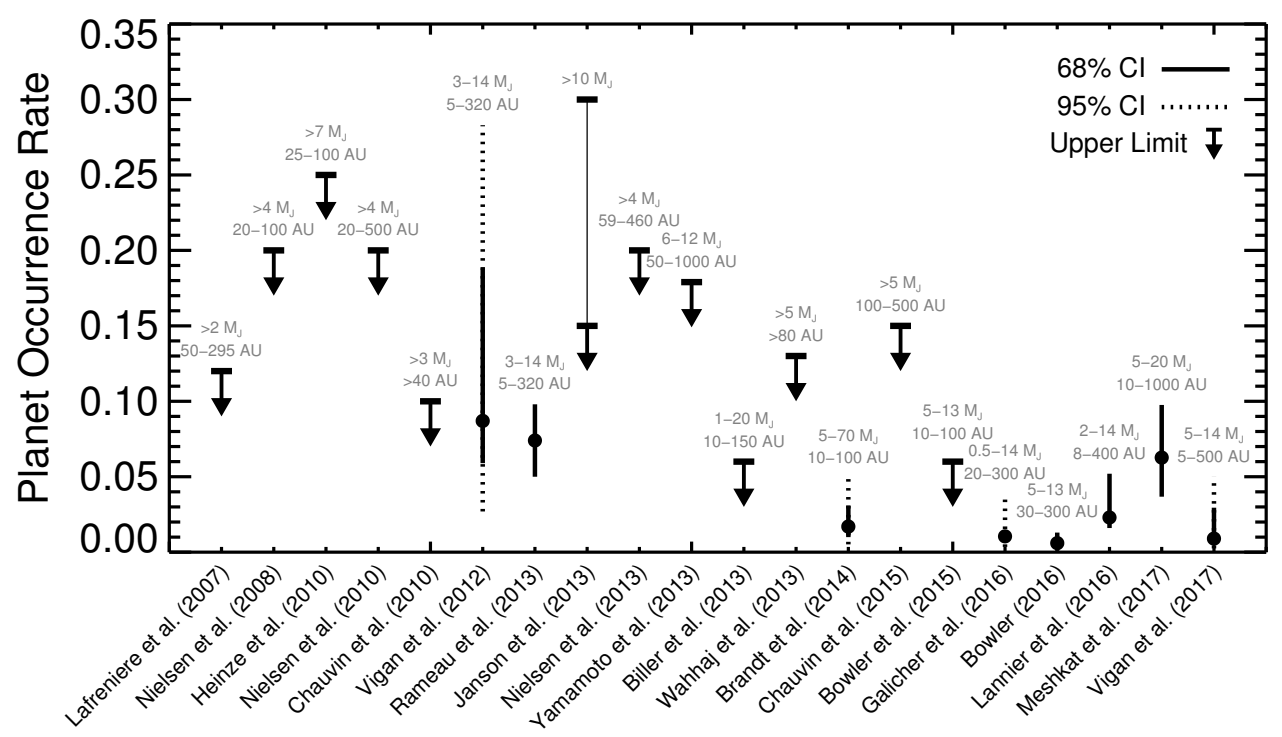

Fig. 2 Occurrence rate measurements of giant planets from direct imaging surveys over the past decade. Most surveys have reported upper limits, but larger meta-analyses over the past few years are converging on a frequency of about $1 \%$ averaged over all host star spectral types. Arrows indicate upper limits with horizontal bars representing the upper limit value. For measurements (as opposed to upper limits), solid and dotted lines denote $68 \%$ and $95 \%$ credible intervals, respectively. Note that these surveys are not all independent; targets may overlap and many surveys incorporate previously published results into their statistical analysis.

The first large-scale compilation of direct imaging surveys was carried out by Nielsen et al. (2008) and expanded to over 118 stars in Nielsen and Close (2010), who measured an upper limit of $<20 \%$ on the frequency of $>4 M_{\text {Jup }}$ planets between $\approx 30-500 \mathrm{AU}$ around Sun-like stars (at the $95 \%$ confidence level). The next major milestone for population statistics was carried out by Brandt et al. (2014b). They merged five surveys totaling a sample of 248 unique targets with spectral types from B to mid-M and found that $1.0-3.1 \%$ of stars host 5-70 $M_{\text {Jup }}$ companions between 10-100 AU (at 68\% confidence; the 95\% credible interval spans 0.52 $4.9 \%)$. In addition, they conclude that the substellar companions from these surveys are consistent with a smooth companion mass and separation distribution $(p(M, a)$ $\propto M^{-0.65 \pm 0.60} a^{-0.85 \pm 0.39}$ ), implying that the planetary-mass companions identified in these surveys may represent the low-mass tail of brown dwarfs rather than a separate population of high-mass planets. Recently, Vigan et al. (2017) compiled the largest collection of imaging results focusing on FGK stars. Based on a sample of 199 targets from 12 previous imaging surveys, they derive a frequency of $0.90 \%$ for 5-14 $M_{\text {Jup }}$ companions spanning 5-500 AU (the 68\% credible interval is 0.702.95 ; the $95 \%$ range is $0.25-5.00 \%$ ). For the entire substellar mass range from 5 to 
$75 M_{\text {Jup }}$, they find a somewhat higher occurrence rate of $2.40 \%$ (with a $95 \%$ credible interval of $0.90-6.80 \%)$. By quantitatively comparing their results with population synthesis models of planets formed via disk instability, they lay the groundwork for direct population-level tests of planet formation theory and conclude that this mode of planet formation is probably inefficient at forming giant planets. At present there are no signs that host star multiplicity dramatically impacts the occurrence rates of circumbinary planets on wide orbits compared to single stars. Bonavita et al. (2016) measure a frequency of $1.3 \%$ for companions spanning $2-15 M_{\text {Jup }}$ between $10-500 \mathrm{AU}$ based on 117 close binary systems (their $95 \%$ confidence range spans $0.35-6.85 \%$ ), which is similar to the rate for their control sample of 205 single stars compiled from the literature.

The largest statistical analyses spanning all host star masses were assembled by Bowler (2016) and Galicher et al. (2016). Based on results from nine published surveys totaling 384 unique and single young stars, Bowler (2016) found that the overall occurrence rate of 5-13 $M_{\text {Jup }}$ giant planets spanning $30-300 \mathrm{AU}$ is $0.6_{-0.5}^{+0.7} \%$ ( $68 \%$ credible interval). For an even wider range of separations from 10 to 1000 $\mathrm{AU}$, the occurrence rate is $0.8_{-0.6}^{+1.0} \%$. This is in good agreement with results from Galicher et al. (2016), who find a frequency of $1.05_{-0.7}^{+2.8} \%$ (95\% credible interval) for 0.5-14 $M_{\text {Jup }}$ planets between 20-300 AU based on their sample of 292 stars spanning B stars to M dwarfs. Altogether, the most massive giant planets reside around roughly $1 \%$ of stars in the interval between a few tens to a few hundred AU.

Planetary-mass companions exist at even wider separations with comparably small frequencies. Durkan et al. (2016) carried out an analysis of Spitzer/IRAC observations of young stars and found an upper limit of 9\% for 0.5-13 $M_{\text {Jup }}$ companions between 100-1000 AU. Naud et al. (2017) measure a frequency of $0.84_{-0.66}^{+6.73} \%$ (at $95 \%$ confidence intervals) for 5-13 $\overline{M_{\text {Jup }}}$ companions between 500-5000 $\mathrm{AU}$, similar to the substellar companion frequency at ultra-wide separations estimated by Aller et al. (2013).

With these large samples in hand, the next natural step is to begin searching for correlations with stellar parameters and environment to better understand the context in which this population of planetary-mass companions forms. Several surveys have specifically focused on high-mass stars (Ehrenreich et al. 2010, Janson et al. 2011; Vigan et al. 2012; Nielsen et al. 2013) and low-mass stars (Delorme et al. 2012, Bowler et al. 2015; Lannier et al. 2016) to examine the occurrence rate of giant planets as a function of stellar host mass. Lannier et al. (2016) find intriguing hints of a trend with stellar host mass, but this has not been recovered with larger samples by Bowler (2016) and Galicher et al. (2016). Breaking their sample into spectral type bins, Bowler (2016) measures an occurrence rate of $2.8_{-2.3}^{+3.7} \%$ from 110 young BA stars, $<4.1 \%$ from 155 FGK stars, and $<3.9 \%$ from $119 \mathrm{M}$ dwarfs at separations of 30-300 AU. This is suggestive of a positive correlation with stellar host mass, but larger samples are still needed to unambiguously validate this with statistical rigor.

Following the discovery of the planets orbiting HR 8799 and $\beta$ Pic, several surveys focused their attention on stars hosting debris disks in anticipation of a trend with wide-separation planets (Janson et al. 2013, Rameau et al. 2013a; Wahhaj et al. 2013b; Meshkat et al. 2015a). The best evidence for a correlation with debris disks 
is presented by Meshkat et al. (2017). They assemble a sample of new and published results of 130 single stars hosting debris disks and compare this with a control sample of 277 stars, ensuring the age and host star mass distributions are similar. Meshkat et al. measure a planet frequency of $6.3 \%$ for the debris disk sample with a $68 \%$ confidence interval spanning 3.7-9.8\%- which is higher than the control sample with $88 \%$ certitude. While a firm confirmation is needed with larger samples, this is the best evidence for any correlation with host star properties that has emerged thus far with direct imaging.

\section{Occurrence Rate of Brown Dwarf Companions}

Brown dwarfs are more luminous than giant planets at the same age, so brown dwarf companions to stars have naturally been detected in higher numbers than planets in direct imaging surveys. Numerous stars with brown dwarf companions have been identified by surveys geared toward finding exoplanets (e.g., PZ Tel B, Biller et al. 2010, Mugrauer et al. 2010, 1RXS J235133.3+312720 B, Bowler et al. 2012; HD 984 B, Meshkat et al. 2015b; HR 2562 B, Konopacky et al. 2016b; HD 206893 B, Milli et al. 2017). Like giant planets, the overall frequency of brown dwarf companions to stars is relatively low (below $\approx 5 \%$ ), the luminosity of brown dwarfs decreases with age, and $\sim 1$ hour of AO-fed time on large telescopes is usually required for robust PSF subtraction of each star. As a result, the yield of brown dwarf companions from direct imaging surveys is relatively low compared to stellar companions, but rather high compared to giant planets, making detailed determinations of population properties challenging.

The first direct imaging surveys were limited by the modest inner working angles of the early instruments and coronagraph systems, and therefore first detected the "low hanging fruit" of wide-separation brown dwarfs, like GJ 229 B at an angular distance of 7.7" (Nakajima et al. 1995) and HR 7329 B at 4.2" (Lowrance et al. 2000). These initial surveys found low occurrence rates for these long period brown dwarfs: Oppenheimer et al. (2001) surveyed 107 stars and detected a single brown dwarf, GJ 229 B, while Hinz et al. (2002) did not discover any substellar companions in their survey of 66 stars with sensitivities to brown dwarfs between 100 and 1400 AU. A larger sample of 102 stars by McCarthy and Zuckerman (2004) was sensitive to planetary-mass companions between 75 and $300 \mathrm{AU}$, and a further 178 stars where brown dwarf companions between 140 and $1150 \mathrm{AU}$ could be detected. Only the single candidate substellar companion GL 577 B was discovered but was later shown to be a low-mass star (Mugrauer et al. 2004). These low detection rates made a robust measurement of the substellar occurrence rate difficult.

Later surveys focused on younger targets and utilized instruments capable of imaging brown dwarfs much closer to the host star (to within $\sim 1^{\prime \prime}$ ), and could thus reach a broader population of brown dwarfs. With larger samples and greater sensitivity, these studies were better able to directly measure the frequency of brown dwarf companions. Metchev and Hillenbrand (2009) surveyed 266 stars, including 
a deep sample of 100 stars with two detections, resulting in a brown dwarf companion frequency of $3.2_{-2.7}^{+3.1} \%$ between $13-75 \mathrm{M}_{\mathrm{Jup}}$ and 28-1590 AU. A similar value was measured by Brandt et al. (2014b) based on giant planets and brown dwarfs discovered by the SEEDS program and several previous surveys. Similarly, Vigan et al. (2017) find a frequency of $2.45 \%$ for 5-75 $M_{\text {Jup }}$ companions between 5-500 $\mathrm{AU}$ (the $95 \%$ credible interval is $0.90-6.95 \%$ ). This occurrence rate appears to be largely independent of age: Ireland et al. (2011) estimate a frequency of $\approx 4 \%$ for substellar companions between $\sim 200-500 \mathrm{AU}$, Lafrenière et al. (2014) measure a substellar frequency of $4.0_{-1.2}^{+3.0} \%$ for 5-40 $M_{\mathrm{Jup}}$ companions between $250-1000 \mathrm{AU}$, Uyama et al. (2017) found that two out of 68 stars younger than 10 Myr host brown dwarfs, and Cheetham et al. (2015) measure a frequency of $7_{-5}^{+8} \%$ for brown dwarfs between 1.3 and $780 \mathrm{AU}$ around $\sim 2 \mathrm{Myr} \rho$ Ophiuchus members. Interestingly, the analysis by Lafrenière et al. (2014) suggests that brown dwarf companions are more common at wider separations ( $>250 \mathrm{AU})$ with $88 \%$ credibility.

Brown dwarf companion frequencies appear to be similar across a broad range of spectral types. $70 \mathrm{~B}$ and A stars imaged by the NICI Campaign (Nielsen et al. 2013) resulted in three brown dwarfs around these stars, while Bowler et al. (2015) observed a similar yield from the PALMS survey of M stars: 4 brown dwarfs out of 78 single stars $\left(2.8_{-1.5}^{+2.4} \%\right.$ for $13-75 M_{\text {Jup }}$ companions between 10-100 AU). Lannier et al. (2016) combined a 58-star VLT/NACO imaging survey with literature results, and found that while there is tentative evidence for a higher frequency of low mass companions around higher mass stars for companions with mass ratios less than 0.01 , for intermediate mass ratios of $0.01-0.05$ (corresponding to brown dwarf masses between 10 and $52 \mathrm{M}_{J u p}$ for a solar mass primary) there is no evidence for a stellar mass dependence. Larger sample sizes will be required to robustly determine the extent to which stellar mass impacts the frequency of brown dwarf companions.

With increasing sample sizes and overall number of discoveries, it is possible to move beyond simply measuring occurrence rates of brown dwarfs to also measure the form of the companion mass function. Brandt et al. (2014a) found a single power law fit masses between 5 and $70 M_{\text {Jup }}$, based on seven detected substellar companions in their sample. In contrast, Reggiani et al. (2016) fit a two-population model to results from the VLT/NACO Large Program and the literature, with overlapping planet and stellar companion distributions giving a minimum value in frequency between 10 and $40 M_{\text {Jup }}$.

The in-progress Gemini Planet Imager Exoplanet Survey (GPIES, Macintosh et al.2015) and SpHere INfrared survey for Exoplanets (SHINE, Feldt et al.|2017) are both targeting $>500$ stars with next-generation imagers capable of detecting brown dwarfs even closer to their parent stars. Already these programs have detected new brown dwarfs around HR 2562 (Konopacky et al.|2016b) and HD 206893 (as part of the SHARDDS campaign, Milli et al.|2017); the final surveys will be well-placed to make a more robust measurement of substellar occurrence rate and determine the nature of the stellar mass and companion mass distributions. 


\section{Conclusions}

Substantial progress has been made over the past decade constraining the statistical properties of giant planets and brown dwarf companions via direct imaging. Altogether, averaged across all host star spectral types (B stars to $\mathrm{M}$ dwarfs), the most precise measurements of the giant planet occurrence rate spanning masses of $\approx 5-$ $13 M_{\text {Jup }}$ for the entire range of separations accessible to direct imaging $(\approx 5-500$ $\mathrm{AU}$ ) is converging to a value near $1 \%$. More nuanced correlations are just beginning to be explored and there are initial indications that giant planets positively scale with the presence of debris disks, and (to a lesser extent) host star mass.

The frequency of brown dwarf companions (13-75 $M_{\text {Jup }}$ ) is consistently found to be between $\approx 1-4 \%$ - albeit with considerable uncertainty among individual measurements - with no obvious signs that this value evolves with age or as a function of stellar host mass. This indicates that the frequency of brown dwarf companions is comparable to those of giant planets, with hints that brown dwarfs may exceed planets by a factor of a few.

There remain many open questions that will be especially suitable to examine when the large (>500 star) surveys with second-generation AO instruments conclude, and in the longer-term with the 30-m class of ground-based extremely large telescopes. These include refining the functional form of the companion mass function and companion mass ratio distribution; more robust tests for correlations with stellar host mass, the presence of debris disks, and multiplicity; searching for signs of evolution in the overall occurrence rate or outer separation cutoff over time; a better understanding of planet multiplicity at wide separations; and eventually completing the bridge between the radial velocity and direct imaging planet distribution functions. The steady improvement of occurrence rate measurements and populations statistics is a testament to the communal effort required to build ever-larger samples and address increasingly rich questions about planet formation, architectures, and evolution.

\section{References}

Aller KM, Kraus AL, Liu MC et al. (2013) A PAN-STARRS + UKIDSS SEARCH FOR YOUNG, WIDE PLANETARY-MASS COMPANIONS IN UPPER SCORPIUS. The Astrophysical Journal 773(1):63

Baraffe I, Chabrier G, Barman TS, Allard F Hauschildt PH (2003) Evolutionary models for cool brown dwarfs and extrasolar giant planets. The case of HD 209458. A\&A 402:701

Baraffe I, Homeier D, Allard F Chabrier G (2015) New evolutionary models for pre-main sequence and main sequence low-mass stars down to the hydrogen-burning limit. A\&A 577:A42

Bate MR, Bonnell IA Bromm V (2003) The formation of a star cluster: predicting the properties of stars and brown dwarfs. MNRAS 339:577

Best WMJ, Liu MC, Dupuy TJ Magnier EA (2017) The Young L Dwarf 2MASS J111932541137466 Is a Planetary-mass Binary . The Astrophysical Journal Letters 843(1):L4

Beust H, Augereau JC, Bonsor A et al. (2014) An independent determination of Fomalhaut b's orbit and the dynamical effects on the outer dust belt. A\&A 561:A43 
Biller BA, Close LM, Masciadri E et al. (2007) An Imaging Survey for Extrasolar Planets around 45 Close, Young Stars with the Simultaneous Differential Imager at the Very Large Telescope and MMT. The Astrophysical Journal Supplement Series 173:143

Biller BA, Liu MC, Wahhaj Z et al. (2010) THE GEMINI NICI PLANET-FINDING CAMPAIGN: DISCOVERY OF A CLOSE SUBSTELLAR COMPANION TO THE YOUNG DEBRIS DISK STAR PZ Tel. The Astrophysical Journal 720(1):L82-L87

Biller BA, Liu MC, Wahhaj Z et al. (2013) THE GEMINI/NICI PLANET-FINDING CAMPAIGN: THE FREQUENCY OF PLANETS AROUND YOUNG MOVING GROUP STARS. ApJ $777(2): 160$

Blunt S, Nielsen EL, De Rosa RJ et al. (2017) Orbits for the Impatient: A Bayesian Rejectionsampling Method for Quickly Fitting the Orbits of Long-period Exoplanets. The Astronomical Journal 153:229

Bonavita M, Chauvin G, Desidera S et al. (2012) MESS (multi-purpose exoplanet simulation system). A\&A 537:A67

Bonavita M, de Mooij EJW Jayawardhana R (2013) Quick-MESS: A fast statistical tool for Exoplanet Imaging Surveys. PASP p 849

Bonavita M, Desidera S, Thalmann C et al. (2016) SPOTS: The Search for Planets Orbiting Two Stars. A\&A 593:A38

Bowler BP (2016) Imaging Extrasolar Giant Planets. Publications of the Astronomical Society of the Pacific 128(968):102,001

Bowler BP, Liu MC, Shkolnik EL et al. (2012) PLANETS AROUND LOW-MASS STARS (PALMS). I. A SUBSTELLAR COMPANION TO THE YOUNG M DWARF 1RXS J235133.3+312720. The Astrophysical Journal 753(2):142

Bowler BP, Liu MC, Shkolnik EL Tamura M (2015) PLANETS AROUND LOW-MASS STARS (PALMS). IV. THE OUTER ARCHITECTURE OF M DWARF PLANETARY SYSTEMS. The Astrophysical Journal Supplement Series 216(1):7

Bowler BP, Liu MC, Mawet D et al. (2017) PLANETS AROUND LOW-MASS STARS (PALMS). VI. DISCOVERY OF A REMARKABLY RED PLANETARY- MASS COMPANION TO THE AB DOR MOVING GROUP CANDIDATE 2MASS J22362452+4751425 . The Astronomical Journal 153(1):1-15

Brandt TD, McElwain MW, Turner EL et al. (2013) NEW TECHNIQUES FOR HIGHCONTRAST IMAGING WITH ADI: THE ACORNS-ADI SEEDS DATA REDUCTION PIPELINE. The Astrophysical Journal 764(2):183

Brandt TD, Kuzuhara M, McElwain MW et al. (2014a) THE MOVING GROUP TARGETS OF THE SEEDS HIGH-CONTRAST IMAGING SURVEY OF EXOPLANETS AND DISKS: RESULTS AND OBSERVATIONS FROM THE FIRST THREE YEARS. ApJ 786(1):1

Brandt TD, McElwain MW, Turner EL et al. (2014b) A STATISTICAL ANALYSIS OF SEEDS AND OTHER HIGH-CONTRAST EXOPLANET SURVEYS: MASSIVE PLANETS OR LOW-MASS BROWN DWARFS? ApJ 794(2):159

Cantalloube F, Mouillet D, Mugnier LM et al. (2015) Direct exoplanet detection and characterization using the ANDROMEDA method: Performance on VLT/NaCo data. A\&A 582:A89

Chauvin G, Lagrange AM, Dumas C et al. (2005) Giant planet companion to 2MASSWJ1207334393254. A\&A 438(2):L25-L28

Chauvin G, Lagrange AM, Bonavita M et al. (2010) Deep imaging survey of young, nearby austral stars. A\&A 509:A52

Chauvin G, Vigan A, Bonnefoy M et al. (2015) The VLT/NaCo large program to probe the occurrence of exoplanets and brown dwarfs at wide orbits. A\&A 573:A127

Chauvin G, Desidera S, Lagrange AM et al. (2017) Discovery of a warm, dusty giant planet around HIP65426. A\&A 605:L9

Cheetham AC, Kraus AL, Ireland MJ et al. (2015) MAPPING THE SHORES OF THE BROWN DWARF DESERT. IV. OPHIUCHUS . ApJ 813(2):1-12

Clanton C Gaudi BS (2016) SYNTHESIZING EXOPLANET DEMOGRAPHICS: A SINGLE POPULATION OF LONG-PERIOD PLANETARY COMPANIONS TO M DWARFS CON- 
SISTENT WITH MICROLENSING, RADIAL VELOCITY,AND DIRECT IMAGING SURVEYS . ApJ 819(2):1-42

Currie T, Muto T, Kudo T et al. (2014) RECOVERY OF THE CANDIDATE PROTOPLANET HD 100546 b WITH GEMINI/NICI AND DETECTION OF ADDITIONAL (PLANETINDUCED?) DISK STRUCTURE AT SMALL SEPARATIONS. The Astrophysical Journal Letters 796(2):L30

De Rosa RJ, Nielsen EL, Blunt SC et al. (2015) ASTROMETRIC CONFIRMATION AND PRELIMINARY ORBITAL PARAMETERS OF THE YOUNG EXOPLANET 51 ERIDANI $b$ WITH THE GEMINI PLANET IMAGER . The Astrophysical Journal Letters 814(1):1-7

Delorme P, Lagrange AM, Chauvin G et al. (2012) High-resolution imaging of young M-type stars of the solar neighbourhood: probing for companions down to the mass of Jupiter. A\&A 539:A72

Delorme P, Gagné J, Girard JH et al. (2013) Direct-imaging discovery of a 12-14 Jupiter-mass object orbiting a young binary system of very low-mass stars. A\&A 553:L5

Durisen RH, Boss AP, Mayer L et al. (2007) Gravitational Instabilities in Gaseous Protoplanetary Disks and Implications for Giant Planet Formation. in Protostars and Planets V, e B Reipurth, D Jewitt, \& K Keil (Tucson, AZ: Univ Arizona Press) p 607

Durkan S, Janson M Carson JC (2016) High Contrast Imaging with Spitzer: Constraining the Frequency of Giant Planets out to 1000 au Separations. The Astrophysical Journal 824(1):58

Ehrenreich D, Lagrange AM, Montagnier G et al. (2010) Deep infrared imaging of close companions to austral A- and F-type stars. A\&A 523:A73

Feldt M, Olofsson J, Boccaletti A et al. (2017) SPHERE/SHINE reveals concentric rings in the debris disk of HIP 73145. A\&A 601:A7

Fortney JJ, Marley MS, Saumon D Lodders K (2008) Synthetic Spectra and Colors of Young Giant Planet Atmospheres: Effects of Initial Conditions and Atmospheric Metallicity. The Astrophysical Journal 683:1104

Galicher R, Marois C, Macintosh B et al. (2016) The International Deep Planet Survey. A\&A 594:A63

Heinze AN, Hinz PM, Kenworthy M et al. (2010) CONSTRAINTS ON LONG-PERIOD PLANETS FROM ANL- ANDM-BAND SURVEY OF NEARBY SUN-LIKE STARS: MODELING RESULTS. The Astrophysical Journal 714(2):1570-1581

Hinz JL, McCarthy DW, Simons DA et al. (2002) A Near-Infrared, Wide-Field, Proper-Motion Search for Brown Dwarfs. The Astronomical Journal 123:2027

Ireland MJ, Kraus A, Martinache F, Law N Hillenbrand LA (2011) Two Wide Planetary-mass Companions to Solar-type Stars in Upper Scorpius. The Astrophysical Journal 726:113

Janson M, Bonavita M, Klahr H et al. (2011) HIGH-CONTRAST IMAGING SEARCH FOR PLANETS AND BROWN DWARFS AROUND THE MOST MASSIVE STARS IN THE SOLAR NEIGHBORHOOD. The Astrophysical Journal 736(2):89

Janson M, Brandt TD, Moro-Martín A et al. (2013) THE SEEDS DIRECT IMAGING SURVEY FOR PLANETS AND SCATTERED DUST EMISSION IN DEBRIS DISK SYSTEMS. ApJ 773(1):73

Jensen-Clem R, Mawet D, Gonzalez CAG et al. (2018) A New Standard for Assessing the Performance of High Contrast Imaging Systems. The Astronomical Journal 155(1):19

Kalas P, Graham JR, Fitzgerald MP Clampin M (2013) STIS CORONAGRAPHIC IMAGING OF FOMALHAUT: MAIN BELT STRUCTURE AND THE ORBIT OF FOMALHAUT b. ApJ 775(1):56

Konopacky QM, Marois C, Macintosh BA et al. (2016a) ASTROMETRIC MONITORING OF THE HR 8799 PLANETS: ORBIT CONSTRAINTS FROM SELF-CONSISTENT MEASUREMENTS . The Astronomical Journal 152(2):1-18

Konopacky QM, Rameau J, Duchene G et al. (2016b) DISCOVERY OF A SUBSTELLAR COMPANION TO THE NEARBY DEBRIS DISK HOST HR 2562. The Astrophysical Journal Letters 829(1): $1-7$

Kratter K Lodato G (2016) Gravitational Instabilities in Circumstellar Disks. Annu Rev Astro Astrophys 54(1):271-311 
Lafrenière D, Doyon R, Marois C et al. (2007a) The Gemini Deep Planet Survey. The Astrophysical Journal 670:1367

Lafrenière D, Marois C, Doyon R, Nadeau D Artigau É (2007b) A New Algorithm for Point-Spread Function Subtraction in High-Contrast Imaging: A Demonstration with Angular Differential Imaging. The Astrophysical Journal 660:770

Lafrenière D, Jayawardhana R, van Kerkwijk MH, Brandeker A Janson M (2014) AN ADAPTIVE OPTICS MULTIPLICITY CENSUS OF YOUNG STARS IN UPPER SCORPIUS. ApJ 785(1):47

Lagrange AM, Bonnefoy M, Chauvin G et al. (2010) A Giant Planet Imaged in the Disk of the Young Star Beta Pictoris: Supporting Online Material. Science 329(5987):57-59

Lambrechts M Johansen A (2012) Rapid growth of gas-giant cores by pebble accretion. A\&A 544:A32

Lannier J, Delorme P, Lagrange AM et al. (2016) MASSIVE: A Bayesian analysis of giant planet populations around low-mass stars. A\&A 596:A83

Liu MC, Magnier EA, Deacon NR et al. (2013) THE EXTREMELY RED, YOUNG L DWARF PSO J318.5338-22.8603: A FREE-FLOATING PLANETARY-MASS ANALOG TO DIRECTLY IMAGED YOUNG GAS-GIANT PLANETS. The Astrophysical Journal 777(2):L20

Lowrance PJ, Schneider G, Kirkpatrick JD et al. (2000) A Candidate Substellar Companion to HR 7329. The Astrophysical Journal 541:390

Macintosh B, Graham JR, Ingraham P et al. (2014) First light of the Gemini Planet Imager. Proceedings of the National Academy of Sciences 111(35):12,661-12,666

Macintosh B, Graham JR, Barman T et al. (2015) Discovery and spectroscopy of the young Jovian planet 51 Eri b with the Gemini Planet Imager. Science

Marois C, Macintosh B, Barman T et al. (2008) Direct Imaging of Multiple Planets Orbiting the Star HR 8799. Science 322(5906): 1348-1352

Marois C, Macintosh B Véran JP (2010) Exoplanet imaging with LOCI processing: photometry and astrometry with the new SOSIE pipeline. Proc SPIE 7736:77,361J

Marois C, Correia C, Galicher R et al. (2014) GPI PSF subtraction with TLOCI: the next evolution in exoplanet/disk high-contrast imaging. In: Marchetti E, Close LM Veran JP (eds) SPIE Astronomical Telescopes + Instrumentation, SPIE, p 91480U

Mawet D, Milli J, Wahhaj Z et al. (2014) FUNDAMENTAL LIMITATIONS OF HIGH CONTRAST IMAGING SET BY SMALL SAMPLE STATISTICS. ApJ 792(2):97

McCarthy C Zuckerman B (2004) The Brown Dwarf Desert at 75-1200 AU. The Astronomical Journal 127:2871

Meshkat T, Bailey VP, Su KYL et al. (2015a) SEARCHING FOR PLANETS IN HOLEY DEBRIS DISKS WITH THE APODIZING PHASE PLATE. ApJ 800(1):5

Meshkat T, Bonnefoy M, Mamajek EE et al. (2015b) Discovery of a low-mass companion to the F7V star HD 984. Monthly Notices RAS 453(3):2379-2387

Meshkat T, Mawet D, Bryan ML et al. (2017) A Direct Imaging Survey of Spitzer-detected Debris Disks: Occurrence of Giant Planets in Dusty Systems . The Astronomical Journal 154(6):0-0

Metchev SA Hillenbrand LA (2009) THE PALOMAR/KECK ADAPTIVE OPTICS SURVEY OF YOUNG SOLAR ANALOGS: EVIDENCE FOR A UNIVERSAL COMPANION MASS FUNCTION. The Astrophysical Journal Supplement Series 181(1):62-109

Meyer MR, Amara A, Reggiani M Quanz SP (2017) M Dwarf Exoplanet Surface Density Distribution: A Log-Normal Fit from 0.07-400 AU. arXiv

Milli J, Hibon P, Christiaens V et al. (2017) Discovery of a low-mass companion inside the debris ring surrounding the F5V star HD206893. A\&A 597:L2

Mugrauer M, Neuh User R, Guenther EW et al. (2004) HD?77407 and GJ?577: Two new young stellar binaries. A\&A 417(3):1031-1038

Mugrauer M, Vogt N, Neuhäuser R Schmidt TOB (2010) Direct detection of a substellar companion to the young nearby star PZ Telescopii. A\&A 523:L1

Nakajima T, Oppenheimer BR, Kulkarni SR et al. (1995) Discovery of a Cool Brown Dwarf. Nature 378(6556):463-465 
Naud ME, Artigau E, Doyon R et al. (2017) PSYM-WIDE: A Survey for Large-separation Planetary-mass Companions to Late Spectral Type Members of Young Moving Groups. The Astronomical Journal 154:154-129

Nielsen EL Close LM (2010) A UNIFORM ANALYSIS OF 118 STARS WITH HIGHCONTRAST IMAGING: LONG-PERIOD EXTRASOLAR GIANT PLANETS ARE RARE AROUND SUN-LIKE STARS. The Astrophysical Journal 717(2):878-896

Nielsen EL, Close LM, Biller BA, Masciadri E Lenzen R (2008) Constraints on Extrasolar Planet Populations from VLT NACO/SDI and MMT SDI and Direct Adaptive Optics Imaging Surveys: Giant Planets are Rare at Large Separations. The Astrophysical Journal 674:466

Nielsen EL, Liu MC, Wahhaj Z et al. (2013) THE GEMINI NICI PLANET-FINDING CAMPAIGN: THE FREQUENCY OF GIANT PLANETS AROUND YOUNG B AND A STARS. ApJ 776(1):4

Nielsen EL, Liu MC, Wahhaj Z et al. (2014) THE GEMINI NICI PLANET-FINDING CAMPAIGN: THE ORBIT OF THE YOUNG EXOPLANET $\beta$ PICTORIS b. ApJ 794(2):158

Oppenheimer BR, Golimowski DA, Kulkarni SR et al. (2001) A Coronagraphic Survey for Companions of Stars within 8 Parsecs. The Astronomical Journal 121:2189

Pueyo L (2016) DETECTION AND CHARACTERIZATION OF EXOPLANETS USING PROJECTIONS ON KARHUNEN-LOEVE EIGENIMAGES: FORWARD MODELING . ApJ 824(2):1-29

Pueyo L, Crepp JR, Vasisht G et al. (2012) APPLICATION OF A DAMPED LOCALLY OPTIMIZED COMBINATION OF IMAGES METHOD TO THE SPECTRAL CHARACTERIZATION OF FAINT COMPANIONS USING AN INTEGRAL FIELD SPECTROGRAPH. The Astrophysical Journal Supplement Series 199(1):6

Rameau J, Chauvin G, Lagrange AM et al. (2013a) A survey of young, nearby, and dusty stars conducted to understand the formation of wide-orbit giant planets. A\&A 553:A60

Rameau J, Chauvin G, Lagrange AM et al. (2013b) CONFIRMATION OF THE PLANET AROUND HD 95086 BY DIRECT IMAGING. The Astrophysical Journal 779(2):L26

Rameau J, Nielsen EL, De Rosa RJ et al. (2016) CONSTRAINTS ON THE ARCHITECTURE OF THE HD 95086 PLANETARY SYSTEM WITH THE GEMINI PLANET IMAGER . The Astrophysical Journal Letters 822(2):1-7

Reggiani M, Meyer MR, Chauvin G et al. (2016) The VLT/NaCo large program to probe the occurrence of exoplanets and brown dwarfs at wide orbits. A\&A 586:A147

Ruffio JB, Macintosh B, Wang JJ et al. (2017) Improving and Assessing Planet Sensitivity of the GPI Exoplanet Survey with a Forward Model Matched Filter. arXiv (1):14

Samland M, Mollière P, Bonnefoy M et al. (2017) Spectral and atmospheric characterization of 51 Eridani $\mathrm{b}$ using VLT/SPHERE. A\&A 603:A57

Soummer R, Pueyo L Larkin J (2012) DETECTION AND CHARACTERIZATION OF EXOPLANETS AND DISKS USING PROJECTIONS ON KARHUNEN-LOĖVE EIGENIMAGES. The Astrophysical Journal 755(2):L28

Todorov K, Luhman KL Mcleod KK (2010) DISCOVERY OF A PLANETARY-MASS COMPANION TO A BROWN DWARF IN TAURUS. The Astrophysical Journal 714(1):L84-L88

Uyama T, Hashimoto J, Kuzuhara M et al. (2017) The SEEDS High-Contrast Imaging Survey of Exoplanets Around Young Stellar Objects. The Astronomical Journal 153(3):1-27

Veras D, Crepp JR Ford EB (2009) Formation, Survival, and Detectability of Planets Beyond 100 AU. The Astrophysical Journal 696:1600

Vigan A, Patience J, Marois C et al. (2012) The International Deep Planet Survey. I. The frequency of wide-orbit massive planets around A-stars. A\&A 544:9

Vigan A, Bonavita M, Biller B et al. (2017) The VLT/NaCo large program to probe the occurrence of exoplanets and brown dwarfs at wide orbits. A\&A 603:A3

Wagner K, Apai D, Kasper M et al. (2016) Direct imaging discovery of a Jovian exoplanet within a triple-star system. Science

Wahhaj Z, Liu MC, Biller BA et al. (2013a) THE GEMINI NICI PLANET-FINDING CAMPAIGN: THE COMPANION DETECTION PIPELINE. ApJ 779(1):80 
Wahhaj Z, Liu MC, Nielsen EL et al. (2013b) THE GEMINI PLANET-FINDING CAMPAIGN: THE FREQUENCY OF GIANT PLANETS AROUND DEBRIS DISK STARS. The Astrophysical Journal 773(2):179

Wahhaj Z, Cieza LA, Mawet D et al. (2015) Improving signal-to-noise in the direct imaging of exoplanets and circumstellar disks with MLOCI. A\&A 581:A24

Wang JJ, Graham JR, Pueyo L et al. (2016) THE ORBIT AND TRANSIT PROSPECTS FOR $\beta$ PICTORIS b CONSTRAINED WITH ONE MILLIARCSECOND ASTROMETRY. The Astronomical Journal 152(4):1-16

Wertz O, Absil O, Gomez Gonzalez CA et al. (2017) VLT/SPHERE robust astrometry of the HR8799 planets at milliarcsecond-level accuracy. A\&A 598:A83

Yamamoto K, Matsuo T, Shibai H et al. (2013) Direct Imaging Search for Extrasolar Planets in the Pleiades. PASJ 